\title{
Noncommutative Bayesian Statistical Inference from a wedge of a Bifurcate Killing Horizon
}

\author{
Gavriel Segre*
}

(Dated: 25-4-2004)

\begin{abstract}
Expanding the remark5.2.7 of [1] the noncommutative bayesian statistical inference from one wedge of a bifurcate Killing horizon is analyzed looking at its inter-relation with the Unruh effect
\end{abstract}

${ }^{*}$ URL: http://www.gavrielsegre.com Electronic address: info@gavrielsegre.com 


\section{Contents}

I. Notation

II. Noncommutative Bayesian Statistical Inference

III. Noncommutative Statistical inference from a wedge of a bifurcate Killing horizon7

IV. The Minkowski case

V. The De Sitter case 


\section{NOTATION}

\begin{tabular}{|c|c|}
\hline$x=y$ & $\mathrm{x}$ is equal to $\mathrm{y}$ \\
\hline$x:=y$ & $\mathrm{x}$ is defined as $\mathrm{y}$ \\
\hline $\operatorname{card}(S)$ & cardinality of $\mathrm{S}$ \\
\hline$f_{\star}$ & differential map of $f$ \\
\hline$f^{\star}$ & pull-back of $\mathrm{f}$ \\
\hline $\mathcal{L}_{X}$ & Lie derivative w.r.t. $\mathrm{X}$ \\
\hline$I s\left[\left(M, g_{a b}\right)\right]$ & isometry group of the space-time $\left(M, g_{a b}\right)$ \\
\hline$\Gamma\left(T^{(r, s)} M\right)$ & sections of the $(\mathrm{r}, \mathrm{s})$-tensor bundle over $\mathrm{M}$ \\
\hline$I^{-}(S)$ & chronological past of the space-time's region $\mathrm{S}$ \\
\hline$I^{+}(S)$ & chronological future of the space-time's region $\mathrm{S}$ \\
\hline$J^{-}(S)$ & causal past of the space-time's region $\mathrm{S}$ \\
\hline$J^{+}(S)$ & causal future of the space-time's region $\mathrm{S}$ \\
\hline$D^{-}(S)$ & past domain of dependence of the space-time's region $S$ \\
\hline$D^{+}(S)$ & future domain of dependence of the space-time's region $\mathrm{S}$ \\
\hline$D(S)$ & domain of dependence of the space-time's region $\mathrm{S}$ \\
\hline$S(A)$ & space of the states over a $W^{\star}$-algebra \\
\hline$\tau_{\text {unbiased }}$ & unbiased state \\
\hline$A_{\left(M, g_{a b}\right)}^{W}$ & Weyl algebra of the space-time $\left(M, g_{a b}\right)$ \\
\hline$S_{H}\left(A_{\left(M, g_{a b}\right)}^{W}\right)$ & Hadamard states over $A_{\left(M, g_{a b}\right)}^{W}$ \\
\hline$\sigma_{t}^{\omega}$ & modular group of the state $\omega$ \\
\hline$A U T(A)$ & automorphisms of the $W^{\star}$-algebra A \\
\hline$I N N(A)$ & inner automorphisms of the $W^{\star}$-algebra A \\
\hline$O U T(A)$ & outer automorphisms of the $W^{\star}$-algebra A \\
\hline$G R-A U T(G, A)$ & automorphisms' groups of A representing G \\
\hline$G R-I N N(G, A)$ & inner automorphisms' groups of A representing G \\
\hline$G R-O U T(G, A)$ & outer automorphisms' groups of A representing G \\
\hline (S) & semi-direct product of groups \\
\hline
\end{tabular}




\section{NONCOMMUTATIVE BAYESIAN STATISTICAL INFERENCE}

Noncommutative Bayesian Statistical Inference, as introduced by Miklos Redei in the $8^{\text {th }}$ chapter "Quantum conditional and quantum conditional probability" of [2], is based on the following analysis.

Given a classical probability space $(X, \sigma, \mu)$ let us suppose to be a statistician having access only to the partial information concerning the probability of an event $B \in \sigma$ and whose goal is to estimate the unknown probability $\mu(A)$ of an arbitrary event $A \in \sigma$.

The Bayesian recipe prescribes that, before using even the partial information he has, the more natural estimation of $\mu(A)$ is the one introducing no bias, i.e.:

$$
\mu_{A P R I O R I}(A):=P_{\text {unbiased }}(A)
$$

i.e. the uniform distribution over $(X, \sigma)$ whether $\operatorname{card}(X)<\aleph_{0}$ or the Lebesgue measure over $(X, \sigma)$ whether $\operatorname{card}(X)=\aleph_{1}$.

Let us observe that in the case $\operatorname{card}(X)=\aleph_{0}$ the unbiased probability measure over $(X, \sigma)$ doesn't exist so that the Bayesian strategy of statistical inference is not defined in that case.

The acquisition of the partial information he can access results, according to the Bayesian recipe, in the following ansatz:

$$
\mu_{A \text { PRIORI }}(A):=P_{\text {unbiased }}(A) \rightarrow \mu_{A \text { POSTERIORI }}(A):=\frac{\mu_{A \text { PRIORI }}(A \bigcap B)}{\mu(B)}
$$

Let us now recall the Basic Theorem of Noncommutative Probability stating that the category having as objects the classical probability spaces and as morphisms their automorphisms is equivalent to the category having as objects the algebraic commutative probability spaces and as morphisms their automorphisms.

Such a theorem naturally leads to a noncommutative generalization of the Bayesian recipe consisting in:

1. the recasting of eq:2.2 in the language of algebraic probability spaces

2. the generalization to noncommutative probability spaces

Given the algebraic commutative probability space $(A, \omega)$ with:

$$
A:=L^{\infty}(X, \sigma, \mu)
$$




$$
\omega(a):=\int_{X} d \mu a \quad a \in A
$$

let us suppose that the statistician has access only to the information concerning a sub- $\sigma$ algebra $\sigma_{\text {accessible }}$ of $\sigma$.

Introduced the $W^{\star}-$ algebra:

$$
A_{\text {accessible }}:=L^{\infty}\left(X, \sigma_{\text {accessible }}, \mu_{\text {accessible }}\right)
$$

where:

$$
\mu_{\text {accessible }}:=\left.\mu\right|_{\sigma_{\text {accessible }}}
$$

and the associated state $\omega_{\text {accessible }} \in S\left(A_{\text {accessible }}\right)$ :

$$
\omega_{\text {accessible }}(a):=\int_{X} d \mu_{\text {accessible }} a \quad a \in A_{\text {accessible }}
$$

we can express the Bayesian recipe in the following way:

1. before using even the partial information that is accessible to him, the better a-priori estimation of $\omega$ the statistician can perform consists in introducing no bias, assuming that:

$$
\omega_{A P R I O R I}:=\tau_{\text {unbiased }}
$$

where $\tau_{\text {unbiased }}$ is the unbiased state over A :

$$
\tau_{\text {unbiased }}(a):=\int_{X} d P_{\text {unbiased }} a \quad a \in A
$$

2. the adoption of the available information may be encoded in the passage from the a-priori estimation to the a-posteriori estimation of $\omega$ specified by the Bayes rule:

$$
\omega_{A \text { PRIORI }}(\cdot)=\tau_{\text {unbiased }}(\cdot) \rightarrow \omega_{\text {A POSTERIORI }}(\cdot):=\omega_{\text {accessible }}\left(E_{\text {A PRIORI }} \cdot\right)
$$

where $E_{A \text { PRIORI }}: A \mapsto A_{\text {accessible }}$ is the conditional expectation w.r.t. $A_{\text {accessible }}$ $\omega_{A} P R I O R I$-invariant

The Basic Theorem of Noncommutative Probability allows to generalize immediately such a recipe to the noncommutative case in which a statistician has access only to the 
sub-noncommutative probability space $\left(A_{\text {accessible }}, \omega_{\text {accessible }}\right)$ of a larger noncommutative probability space $(A, \omega)$ :

$$
\omega_{\text {accessible }}:=\left.\omega\right|_{A_{\text {accessible }}}
$$

resulting in the following noncommutative bayesian recipe:

1. before using even the partial information that is accessible to him, the better a-priori estimation of $\omega$ consists in introducing no bias, assuming that:

$$
\omega_{A P R I O R I}:=\tau_{\text {unbiased }}
$$

where $\tau_{\text {unbiased }}$ is the noncommutative unbiased probability distribution over $A$, namely the tracial state on it.

2. the adoption of the available information may be encoded in the passage from the a-priori estimation to the a-posteriori estimation of $\omega$ specified by the noncommutative Bayes rule:

$$
\omega_{\text {A PRIORI }}(\cdot)=\tau_{\text {unbiased }}(\cdot) \rightarrow \omega_{\text {A POSTERIORI }}(\cdot):=\omega_{\text {accessible }}\left(E_{\text {A PRIORI }} \cdot\right)
$$

where $E_{A \text { PRIORI }}: A \mapsto A_{\text {accessible }}$ is the conditional expectation w.r.t. $A_{\text {accessible }}$ $\omega_{A}$ PRIORI-invariant

Let us now observe that to the feasibility condition for such a statistical inference already present in the commutative case and requiring the existence of the unbiased probability distribution, another constraint has to be added in the noncommutative case: according to Takesaki Theorem $E_{A \text { PRIORI }}$ exists if and only if the following modular constraint is satisfied:

$$
\sigma_{t}^{\omega_{A P R I O R I}}(a) \in A_{\text {accessible }} \forall a \in A_{\text {accessible }}, \forall t \in \mathbb{R}
$$

where $\sigma_{t}^{\omega_{A} \text { PRIORI }}$ denotes the modular group of $\omega_{A \text { PRIORI }}$.

Let us observe, with this regard, that since the modular constraint is not satisfied for any sub- $W^{\star}$-algebra, the philosophical subjectivistic viewpoint consistent in the commutative case, cannot be generalized to the noncommutative case.

What may be generalized to the noncommutative case is the recipe for statistical inference but not "one point of view in its entirety" ${ }^{1}$.

\footnotetext{
${ }^{1}$ citing de Finetti's dedication of his "Theory of Probability" to a Segre I am not, unfortunately, a descendant of
} 


\section{NONCOMMUTATIVE STATISTICAL INFERENCE FROM A WEDGE OF A BIFURCATE KILLING HORIZON}

Given a space-time, i.e. a 4-dimensional lorentzian manifold $\left(M, g_{a b}\right)^{2}$ [3], [4], [5], let us suppose that it admits a bifurcate Killing horizon, i.e. a bidimensional spacelike surface $\mathrm{S}$ such that there exist a Killing vector field $X^{a}$ vanishing on it:

$$
\begin{gathered}
\mathcal{L}_{X^{a}} g_{a b}=0 \\
X^{a}(p)=0 \quad \forall p \in S
\end{gathered}
$$

Let us suppose, furthermore, that $\mathrm{S}$ is a Cauchy surface of $\left(M, g_{a b}\right)^{3}$, i.e. that its domain of dependence is the whole M:

$$
D(S)=M
$$

Denoted by $h_{A}$ and $h_{B}$ the two null surfaces generated by the null geodesics orthogonal to S, M may be expressed as the union of four disjoint wedges:

$$
\begin{aligned}
M & =\cup_{i=1}^{4} W_{i} \\
W_{1} & :=I^{-}\left(h_{A}\right) \cap I^{+}\left(h_{B}\right) \\
W_{2} & :=I^{+}\left(h_{A}\right) \cap I^{-}\left(h_{B}\right) \\
W_{3} & :=J^{+}(S) \\
W_{4} & :=J^{-}(S)
\end{aligned}
$$

Let us now suppose to be a statistician living in a Universe whose Physics sufficentely far from Planck's scale is described by a quantum field theory on $\left(M, g_{a b}\right)$, specified by the set of local observables' algebras $\left\{A_{O}\right\}_{O \subseteq M}$ obeying Dimock's axioms (i.e. Dimock generalization to curved space-time of Haag-Kastler's axioms) [6], [7], 8] whose world-line is a flow line of the above Killing vector field $X^{a}$.

\footnotetext{
${ }^{2}$ I will follow Penrose abstract index notation as explained, e.g., in 3

${ }^{3}$ We have implicitly assumed that $\left(M, g_{a b}\right)$ is globally-hyperbolic and, hence, admits Cauchy surfaces. While in Classical General Relativity the status of the Strong Cosmic Censorship Conjecture stating that any "physical" space-time is globally hyperbolic is dubious, it is strongly dubious whether a Quantum Field Theory on a non globally-hyperbolic space-time may be consistently formalized
} 
Supposing he can access only the state of affairs concerning the physical observables localized in $W_{1}$ his objective is to make a statistical inference concerning the state of affairs outside $W_{1}$.

Denoting by:

$$
A_{\text {accessible }}:=A_{W_{1}}
$$

the algebra of observables that is accessible to him, his objective is to estimate the true state $\omega \in S\left(A_{\left(M, g_{a b}\right)}^{W}\right)$ of the noncommutative probability space $\left(A_{\left(M, g_{a b}\right)}^{W}, \omega\right)$ describing the Universe in the assumed classical-background approximation, $A_{\left(M, g_{a b}\right)}^{W}$ denoting the Weyl algebra of $\left(M, g_{a b}\right)$, from the knowledge of the information accessible to him, codified by the accessible state defined as the restriction of $\omega$ to the accessible algebra:

$$
\omega_{\text {accessible }}:=\left.\omega\right|_{A_{\text {accessible }}} \in S\left(A_{\text {accessible }}\right)
$$

Noncommutative Bayesian Statistical Theory, as described in the previous section, would prescribe to him to adopt the following recipe:

1. before using even the partial information that is accessible to him, the better a-priori estimation of $\omega$ consists in introducing no bias, assuming that:

$$
\omega_{A} \text { PRIORI }:=\tau_{\text {unbiased }}
$$

where $\tau_{\text {unbiased }}$ is the noncommutative unbiased probability distribution over $A_{\left(M, g_{a b}\right)}^{W}$, namely the tracial state on it.

2. the adoption of the available information may be encoded in the passage from the a-priori estimation to the a-posteriori estimation of $\omega$ specified by the noncommutative Bayes rule:

$$
\omega_{\text {A PRIORI }}(\cdot)=\tau_{\text {unbiased }}(\cdot) \rightarrow \omega_{\text {A POSTERIORI }}(\cdot):=\omega_{\text {accessible }}\left(E_{\text {A PRIORI }} \cdot\right)
$$

where $E_{A \text { PRIORI }}: A_{\left(M, g_{a b}\right)}^{W} \mapsto A_{\text {accessible }}$ is the conditional expectation w.r.t. $A_{\text {accessible }} \omega_{A \text { PRIORI-invariant }}$

Let us observe, first of all, that, according to Takesaki Theorem, the existence of the involved conditional expectation and, hence, the feasibility of the Bayesian statistical inference, requires the assumption of the following modular constraint:

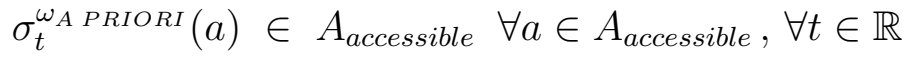


Let us observe, furthermore, that since the Weyl's algebra $A_{\left(M, g_{a b}\right)}^{W}$ of $\left(M, g_{a b}\right)$ is generally not finite, the unbiased noncommutative probability measure $\tau_{\text {unbiased }}$ doesn't exist.

It must be observed, at this point, that there exists, anyway, an a priori information that the statistician can adopt: the fact that the expectation value $\left\langle T_{a b}>\right.$ of the stressenergy operator $T_{a b}$ must be well-defined in order of making the back-reaction's semi-classical Einstein equation:

$$
G_{a b}=8 \pi<T_{a b}>
$$

well-defined too, resulting in the condition that $\omega$ is an Hadamard state over the Weyl's algebra $A_{\left(M, g_{a b}\right)}^{W}$ of $\left(M, g_{a b}\right)$ :

$$
\omega \in S_{H}\left(A_{\left(M, g_{a b}\right)}^{W}\right)
$$

Consequentially it is natural for the statistician to assume that $\omega_{A}$ PRIORI is an Hadamard state too.

$$
\omega_{A P R I O R I} \in S_{H}\left(A_{\left(M, g_{a b}\right)}^{W}\right)
$$

Furthermore he a-priori knows that some information about the observables not accessible to him may be recovered by the information concerning $A_{\text {accessible }}$ through the condition:

$$
\exists\left\{\alpha_{g}\right\} \in G R-I N N\left[I s\left(M, g_{a b}\right), A_{\left(M, g_{a b}\right)}^{W}\right]: \alpha_{g}\left(A_{O}\right)=A_{g} O \quad \forall O \subset W_{1}
$$

Consequentially it is natural, for the statistician, to choose the a-priori state as much $I s\left[\left(M, g_{a b}\right)\right]$-invariant as possible.

To understand how this two constraints concretely work as to the determination of $\omega_{A \text { PRIORI }}$ it is useful to start analyzing the simpler cases. 


\section{THE MINKOWSKI CASE}

Let us start analyzing the simpler particular case in which $\left(M, g_{a b}\right)$ is the Minkowski space-time:

$$
\begin{aligned}
M & :=\mathbb{R}^{4} \\
g_{a b}:=\eta_{a b} & :=\eta_{\mu \nu} d x^{\mu} \otimes d x^{\nu} \\
\eta_{\mu \nu} & :=\operatorname{diag}(-1,1,1,1)
\end{aligned}
$$

The isometries-group of Minkowski space-time is the Poincaré group $S O(1,3)\left(\mathrm{S} \mathbb{R}^{4}\right.$ generated by the 10 Killing vector fields:

$$
\begin{gathered}
T_{(i)}^{\mu}:=\delta_{i}^{\mu} i=0, \cdots, 3 \\
L_{\mu \nu}:=x_{\mu} \partial_{\nu}-x_{\nu} \partial_{\mu} \quad \nu>\mu=0 \cdots, 3
\end{gathered}
$$

Let us then observe that the surface:

$$
S:=\left\{x^{\mu} \in \mathbb{R}^{4}: x^{0}=x^{1}=0\right\}
$$

is a bifurcate Killing horizon for the Killing vector field:

$$
X^{a}:=L_{01}
$$

generating boosts in the direction $x^{1}$. Let us denote by $\alpha_{t}$ the inner automorphisms' group representing the one-dimensional subgroup $i_{t}$ of $I s\left[\left(\mathbb{R}^{4}, \eta_{a b}\right)\right]$ generated by $X^{a}$.

Since the domain of dependence $\mathrm{D}(\mathrm{S})$ of the surface $\mathrm{S}$ is such that:

$$
D(S)=\mathbb{R}^{4}
$$

$\mathrm{S}$ is a Cauchy surface, so that, according to the general analysis previously introduced, one has the splitting of the Minkowski space-time in the four wedges specified by eq 3.4 with:

$$
\begin{aligned}
& h_{A}=\left\{x^{\mu} \in \mathbb{R}^{4}: x^{0}=x^{1}\right\} \\
& h_{B}=\left\{x^{\mu} \in \mathbb{R}^{4}: x^{0}=-x^{1}\right\} \\
& W_{1}=\left\{x^{\mu} \in \mathbb{R}^{4}:\left|x^{1}\right|<x^{0}, x^{0}>0\right\} \\
& W_{2}=\left\{x^{\mu} \in \mathbb{R}^{4}:\left|x^{1}\right|<x^{0}, x^{0}<0\right\} \\
& W_{3}=\left\{x^{\mu} \in \mathbb{R}^{4}:\left|x^{1}\right|>x^{0}, x^{1}>0\right\} \\
& W_{4}=\left\{x^{\mu} \in \mathbb{R}^{4}:\left|x^{1}\right|>x^{0}, x^{1}<0\right\}
\end{aligned}
$$


Since $X^{a}$ is time-like in the two wedges $W_{1}$ and $W_{2}$ its flow $i_{t}$ represent possible world-lines of a massive observer such as our statistician; we will suppose, precisely, that the statistician's world-line is an integral curve of $X^{a}$ contained in $W_{1}$.

Following the condition enunciated in the last section, among the possible Hadamard states that our statistician may choose as a-priori state, the more natural one is the restriction to $A_{W_{1}}$ of the only $I s\left(\mathbb{R}^{4}, \eta_{a b}\right)$-invariant one, i.e. the vacuum state $\omega_{(0)}$ :

$$
\omega_{A P R I O R I}:=\left.\omega_{(0)}\right|_{A_{W_{1}}} \in S\left(A_{W_{1}}\right)
$$

The Unruh effect, consisting in the fact that, in the case $\omega=\omega_{(0)}$ in which the state to estimate is the vacuum one, such a vacuum state appears to the statistician following the flow $i_{t}$ of $L_{01}$ as a thermal bath, has been explained by Geoffrey Sewell in terms of Modular Theory through the Bisognano-Wichmann theorem [9], 7] stating that $\omega_{A P R I O R I}$ is an $\alpha_{t^{-}}$ KMS-state at $\beta=2 \pi$.

Let us now recall that the feasibility of the statistical inferential problem is itself ruled by the modular group of $\omega_{A P R I O R I}$ through the modular constraint of ed 3.12 whose satisfaction, in the present case:

$$
\sigma_{t}^{\omega_{0}}(a) \in A_{W_{1}} \forall a \in A_{W_{1}}
$$

should follows by the $i_{t}$-invariance of $\omega_{0}$, by the fact that $\left(W_{1},\left.\eta_{a b}\right|_{\Gamma\left(T^{\left.(0,2) W_{1}\right)}\right.}\right)$ is a globallyhyperbolic space-time for its own and by the fact that:

$$
\alpha_{t} A_{O}=A_{i_{t} O} \forall O \subset W_{1}
$$

Modular Theory tells us, furthermore, that $\omega_{A \text { PRIORI }}$ is a $\sigma_{-t}^{\omega_{A} \text { PRIORI }}$ - KMS state at $\beta=1$.

This double role of the modular group $\sigma_{t}^{\omega_{A} \text { PRIORI }}$ could suggest an interpretation of the Unruh effect (stating that, in the case $\omega=\omega_{(0)}$ in which the state to estimate is the vacuum one, our accelerated statistician feels a positive temperature) in terms of the Noncommutative Bayesian Statistical Inference he performs about the whole noncommutative probability space $\left(A_{\left(\mathbb{R}^{4}, \eta_{a b}\right)}^{W}, \omega\right)$ having access only to the local information of $A_{W_{1}}$.

Such a strategy of statistical inference is codified through the following modified Bayes recipe:

1. before making use even of the partial information that is accessible to him, the better a-priori estimation of $\omega$ consists in assuming as a-priori-state the restriction of the 
vacuum state to the accessible algebra:

$$
\omega_{A P R I O R I}:=\left.\omega_{(0)}\right|_{A_{W_{1}}}
$$

2. the adoption of the available information may be encoded in the passage from the a-priori estimation to the a-posteriori estimation of $\omega$ specified by the noncommutative Bayes rule:

$$
\omega_{A \text { PRIORI }}(\cdot)=\left.\omega_{(0)}\right|_{A_{W_{1}}}(\cdot) \rightarrow \omega_{A \text { POSTERIORI }}(\cdot):=\omega_{\text {accessible }}\left(E_{\text {A PRIORI }} \cdot\right)
$$

where $E_{A \text { PRIORI }}: A_{\left(\mathbb{R}^{4}, \eta_{a b}\right)}^{W} \mapsto A_{W_{1}}$ is the conditional expectation w.r.t. $A_{W_{1}}$ $\omega_{A}$ PRIORI-invariant 


\section{THE DE SITTER CASE}

Let us then pass to analyze the case in which $\left(M, g_{a b}\right)$ is the De Sitter space-time of unit radius [9]:

$$
\begin{aligned}
M & :=\left\{x^{\mu} \in \mathbb{R}^{5}: \eta_{\mu \nu} x^{\mu} x^{\nu}=1\right\} \\
g_{a b} & :=i^{\star} \eta_{A B} \\
\eta_{A B} & :=\eta_{\mu \nu} d x^{\mu} \otimes d x^{\nu} \\
\eta_{\mu \nu} & :=\operatorname{diag}(-1,1,1,1,1)
\end{aligned}
$$

namely the hyperboloid of unit radius embedded in the (1,4)-Minkowskian space-time $\left(\mathbb{R}^{5}, \eta_{A B}\right)$ endowed with the lorentzian metric induced by the inclusion (identity) embedding $i: M \mapsto \mathbb{R}^{5}: i(p):=p \forall p \in M$

The isometries-group of $\left(\mathbb{R}^{5}, \eta_{A B}\right)$ :

$$
I s\left[\left(\mathbb{R}^{5}, \eta_{A B}\right)\right] \mid=S O(1,4) \mathrm{S} \mathbb{R}^{5}
$$

is generated by the 15 Killing vector fields:

$$
\begin{gathered}
T_{(i)}^{\mu}:=\delta_{i}^{\mu} i=0, \cdots, 4 \\
L_{\mu \nu}:=x_{\mu} \partial_{\nu}-x_{\nu} \partial_{\mu} \quad \nu>\mu=0 \cdots, 4
\end{gathered}
$$

While:

$$
i_{\star} L_{\mu \nu} \in \Gamma(T M) \nu>\mu=0 \cdots, 4
$$

one has that:

$$
i_{\star} T_{(i)}^{\mu} \notin \Gamma(T M) \quad i=0, \cdots, 4
$$

It follows that the isometry group of the De Sitter space-time $\left(M, g_{a b}\right)$ :

$$
\operatorname{Is}\left[\left(M, g_{a b}\right)\right]=S O(1,4)
$$

is generated by the 10 Killing vector fields:

$$
i_{\star} L_{\mu \nu} \in \Gamma(T M) \nu>\mu=0, \cdots, 4
$$


Let us then observe that:

$$
S:=\left\{x^{\mu} \in \mathbb{R}^{5}: x^{0}=x^{1}=0\right\}
$$

is a bifurcate Killing horizon for the Killing vector field $L_{01}$ of $\left(\mathbb{R}^{5}, \eta_{A B}\right)$ generating boosts in the direction $x^{1}$.

Since the domain of dependence $\mathrm{D}(\mathrm{S})$ of the surface $\mathrm{S}$ is such that:

$$
D(S)=\mathbb{R}^{5}
$$

, i.e. $\mathrm{S}$ is a Cauchy surface of $\left(\mathbb{R}^{5}, \eta_{A B}\right)$, according to the general analysis previously introduced one has the splitting of the (1,4)-Minkowski space-time in four wedges specified by eq 3.4 with:

$$
\begin{aligned}
& h_{A}=\left\{x^{\mu} \in \mathbb{R}^{5}: x^{0}=x^{1}\right\} \\
& h_{B}=\left\{x^{\mu} \in \mathbb{R}^{5}: x^{0}=-x^{1}\right\} \\
& W_{1}=\left\{x^{\mu} \in \mathbb{R}^{5}:\left|x^{1}\right|<x^{0}, x^{0}>0\right\} \\
& W_{2}=\left\{x^{\mu} \in \mathbb{R}^{5}:\left|x^{1}\right|<x^{0}, x^{0}<0\right\} \\
& W_{3}=\left\{x^{\mu} \in \mathbb{R}^{5}:\left|x^{1}\right|>x^{0}, x^{1}>0\right\} \\
& W_{4}=\left\{x^{\mu} \in \mathbb{R}^{5}:\left|x^{1}\right|>x^{0}, x^{1}<0\right\}
\end{aligned}
$$

It follows that $\left.S\right|_{M}$ is a bifurcate Killing horizon for the Killing vector field:

$$
X^{a}:=i_{\star} L_{01}
$$

of $\left(M, g_{a b}\right)$. Since $\left.S\right|_{M}$ is a Cauchy surface of $\left(M, g_{a b}\right)$ :

$$
D\left(\left.S\right|_{M}\right)=M
$$

one has the splitting of $\left(M, g_{a b}\right)$ into the four wedges specified by eq 3.4 with:

$$
H_{i}:=\left.W_{i}\right|_{M} \quad i=1, \cdots, 4
$$

Since the $I s\left(M, g_{a b}\right)$-invariance selects again a single state, the vacuum state $\omega_{0}$, among the Hadamard ones, the prescribed conditions for the selection of the a-priori state lead us to the modified Bayes recipe: 
1. before making use even of the partial information that is accessible to him, the better a-priori estimation of $\omega$ consists in assuming as a-priori-state the restriction of the vacuum state to the accessible algebra:

$$
\omega_{A \text { PRIORI }}:=\left.\omega_{(0)}\right|_{A_{H_{1}}}
$$

2. the adoption of the available information may be encoded in the passage from the a-priori estimation to the a-posteriori estimation of $\omega$ specified by the noncommutative Bayes rule:

$$
\omega_{A \text { PRIORI }}(\cdot)=\left.\omega_{(0)}\right|_{A_{H_{1}}}(\cdot) \rightarrow \omega_{\text {A POSTERIORI }}(\cdot):=\omega_{\text {accessible }}\left(E_{\text {A PRIORI }} \cdot\right)
$$

where $E_{A \text { PRIORI }}: A_{\left(M, g_{a b}\right)}^{W} \mapsto A_{W_{1}}$ is the conditional expectation w.r.t. $A_{W_{1}}$ $\omega_{A P R I O R I^{-i n v a r i a n t}}$

Let us denote by $\alpha_{t}$ the inner automorphisms' group representing the one-dimensional subgroup $i_{t}$ of $I s\left[\left(M, g_{a b}\right)\right]$ generated by $X^{a}$.

The Unruh effect, consisting in the fact that, in the case $\omega=\omega_{(0)}$ in which the state to estimate is the vacuum one, such a vacuum state appears to the statistician following the flow of $X^{a}$ as a thermal bath, has been recasted by Figari, H öegh-Krohn an Nappi and later by Bros and Moschella in terms of Modular Theory through the Bisognano-Wichmann theorem [9], 7] stating that $\omega_{\text {APRIORI }}$ is an $\alpha_{t^{-}}$KMS-state at $\beta=2 \pi$.

Let us now recall that, exactly as in the Minkowskian case, the feasibility of the statistical inferential problem is itself ruled by the modular group of $\omega_{A P R I O R I}$ through the modular constraint of ed 3.12 whose satisfaction, in the present case:

$$
\sigma_{t}^{\omega_{0}}(a) \in A_{H_{1}} \forall a \in A_{H_{1}}
$$

should follows by the $i_{t^{-}}$invariance of $\omega_{0}$, by the fact that $\left(H_{1},\left.g_{a b}\right|_{\Gamma\left(T^{\left.(0,2) H_{1}\right)}\right.}\right)$ is a globallyhyperbolic space-time for its own, and by the fact that:

$$
\alpha_{t} A_{O}=A_{i_{t} O} \quad \forall O \subset H_{1}
$$

Modular Theory, furthermore, tells us that $\omega_{A \text { PRIORI }}$ is a $\sigma_{-t}^{\omega_{A} \text { PRIORI }}$ - KMS state at $\beta=1$. So, once again, this double role of the modular group $\sigma_{t}^{\omega_{A} \text { PRIORI }}$ could suggest an interpretation of the Unruh effect in terms of the Noncommutative Bayesian Statistical Inference he performs about the whole noncommutative probability space $\left(A_{\left(M, g_{a b}\right)}^{W}, \omega\right)$ having access only to the local information of $A_{H_{1}}$. 
[1] G. Segre. Algorithmic Information Theoretic Issues in Quantum Mechanics. PhD thesis, Dipartimento di Fisica Nucleare e Teorica. quant-ph/0110018, Pavia (Italy), 2002.

[2] M. Redei. Quantum Logic in Algebraic Approach. Kluwer Academic Publishers, Dordrecht, 1998.

[3] R.M. Wald. General Relativity. The University of Chicago Press, Chicago, 1984.

[4] R.M. Wald. Quantum Field Theory in Curved Spacetime and Black Hole Thermodynamics. The University of Chicago Press, Chicago, 1994.

[5] S. Kobayashi K. Nomizu. Foundation of Differential Geometry. John Wiley and Sons, 1996.

[6] J. Dimock. Algebras of local observables on a manifold. Commun. Math. Phys., 77:219, 1980.

[7] R. Haag. Local Quantum Physics. Fields, Particles, Algebras. Springer-Verlag, New York, 1996.

[8] R. Verch. On Generalizations of the Spectrum Condition. In R. Longo, editor, Mathematical Physics in Mathematics and Physics, pages 409-428. American Mathematical Society, Providence, Rhode Island, 2002.

[9] H. Narnhofer I. Peter W. Thirring. How hot is the De Sitter space? In Selected Papers of W.E. Thirring, pages 613-616. American Mathematical Society, Providence, Rhode Island, 1998. 